\title{
Growth Hormone and Endometrial Receptivity
}

\author{
Signe Altmäe ${ }^{1,2,3 *}$ and Lusine Aghajanova ${ }^{4}$ \\ ${ }^{1}$ Department of Biochemistry and Molecular Biology, Faculty of Sciences, University of Granada, Granada, Spain, \\ ${ }^{2}$ Competence Centre on Health Technologies, Tartu, Estonia, ${ }^{3}$ Instituto de Investigación Biosanitaria ibs. GRANADA, \\ Granada, Spain, ${ }^{4}$ Division of Reproductive Endocrinology and Infertility, Department of Obstetrics and Gynecology, Stanford \\ School of Medicine, Sunnyvale, CA, United States
}

Administration of growth hormone $(\mathrm{GH})$ during ovarian stimulation has shown beneficial effects on in vitro fertilization (IVF) outcomes. It is generally believed that this improvement is due to the stimulating effect of GH on oocyte quality. However, studies are emerging that show possible positive effect of $\mathrm{GH}$ administration on endometrial receptivity, thus suggesting an additional potential benefit at the level of the uterus, especially among women with recurrent implantation failure, thin endometrium, and older normal responders. This review summarizes recent data on $\mathrm{GH}$ co-treatment effects on endometrium and endometrial receptivity among infertile women undergoing IVF, and proposes possible mechanisms of $\mathrm{GH}$ actions in the endometrium.

Keywords: endometrium, endometrial receptivity, growth hormone, infertility, in vitro fertilization, transcriptome

\section{OPEN ACCESS}

Edited by:

John Lui Yovich,

Pivet Medical Center, Australia

Reviewed by:

Biagio Cangiano,

University of Milan, Italy

Nalini Mahajan

Independent Researcher, India

*Correspondence:

Signe Altmäe

signealtmae@ugr.es

Specialty section: This article was submitted to

Reproduction,

a section of the journal

Frontiers in Endocrinology

Received: 09 July 2019 Accepted: 09 September 2019 Published: 24 September 2019

Citation:

Altmäe S and Aghajanova L (2019) Growth Hormone and Endometrial Receptivity. Front. Endocrinol. 10:653.

doi: 10.3389/fendo.2019.00653

\section{INTRODUCTION}

Receptive endometrium is an absolute prerequisite for a successful embryo implantation, being defined by a limited time-frame when the endometrium is favorable for embryo adhesion and the subsequent attachment and invasion processes (1).

Endometrial receptivity is a complex process that is orchestrated by the synergistic actions of main reproductive hormones estrogen and progesterone, as well as plead of other endocrine, paracrine and autocrine factors $(2,3)$. Impaired endometrial receptivity is thought to be one of the major reasons for embryo implantation failure (4). In assisted reproductive technologies (ART), where the good quality embryos are transferred as a standard of care, implantation failure remains an unsolved obstacle $(5,6)$. Regardless of the advances in assisted reproduction, particularly regarding the more effective means of embryo selection and cryopreservation, many patients repeatedly fail the treatment procedure. What we are facing today is that implantation failure in ART is common, and we lack the evidence-based therapeutic solutions for treating it. As a result, clinicians often feel obliged to offer treatments that are largely empirical, based on some biologic rationale but with little clinical evidence to support their use $(7,8)$. The treatment failure is equally frustrating to both patients and their providers, which even more emphasizes the urgent need for novel effective treatment to prevent yet another failure.

The role of growth hormone (GH) in female reproduction has gained renewed interest and has become a heated topic over the last decade. The local GH production in the reproductive tissues themselves exert an important autocrine/intracrine effects on those tissues, in addition to the pituitary production of GH (9). Moreover, local insulin growth factor 1 (IGF-1) production (known downstream mediator of $\mathrm{GH}$ ) has been shown to be controlled by gonadotropins and estradiol as well (10). Evidence emerging from clinical practice suggests that GH administration during ovarian stimulation may improve oocyte quality [higher number of oocytes collected, higher fertilization 
rate, and higher number of embryos reaching the transfer stage (11-15)], increase pregnancy rate (16-24), implantation rate (16, $20-23,25,26)$, and live birth rate $(12,16,19,20,23,25,27)$. The accumulating beneficial effects of GH on assisted reproduction outcomes do not exclude the possibility that this effect is due, at least in part, to an action of GH on endometrial receptivity.

\section{GROWTH HORMONE IN THE ENDOMETRIUM}

$\mathrm{GH}$ is a peptide hormone secreted by the anterior pituitary gland, having important role in cell growth and metabolism throughout the body. GH together with its receptor, GHR, and related growth factors including IGF-1, is expressed in the endometrium of rats and human (28-31). The study by Sbracia et al. obtained biopsies from women in proliferative and secretory phases, as well as first trimester decidua (from elective pregnancy terminations) (28). They showed that there was no $\mathrm{GH}$ expression in proliferative glandular epithelium, but GH immunoreactivity appeared in the mid-luteal secretory phase (no subdivision within secretory phase was done) and increased in the decidua from the first trimester abortions, with similar expression in the decidual samples from the term pregnancies, suggesting a role in embryo implantation process. Interestingly, no stromal expression of $\mathrm{GH}$ was observed in any sample (28). Moreover, the authors analyzed $\mathrm{GH}$ expression in the endometrium from women with "luteal phase defect," defined by low progesterone levels $<8 \mathrm{ng} / \mathrm{mL}$ and delayed endometrial maturation, and saw significantly lower expression of $\mathrm{GH}$ (28). This data suggested close relationship between $\mathrm{GH}$ expression in endometrium and progesterone level/function. Further, a recent study on human endometrial cell line indicated that $\mathrm{GH}$ may act in a direct or IGF-1-mediated manner on human endometrial cells to promote proliferation and vascularization and up-regulation of receptivity-related genes such as vascular endothelial growth factor (VEGF) and integrin beta 3 (ITGB3) (21). VEGF is an important player in angiogenesis (32), and it has been shown to act in an autocrine manner on endometrial epithelial cell adhesion as a key regulator in the implantation process (33). ITGB3 is a well-known biomarker of receptivity (34), and down-regulation of this biomarker (phenomenon detected in women with unexplained infertility, endometriosis, and luteal phase deficiency) has been related to lower pregnancy rates $(35,36)$.

Apart from the effects of circulating $\mathrm{GH}$ and locally produced $\mathrm{GH}$ on endometrium, there is a proposed indirect effect of ovarian GH on endometrial function, namely its involvement in the function and maintenance of the corpus luteum (37, 38). While the majority of the data come from various animal models, they are nevertheless significant. Luteal function and its maintenance are vital for the establishment of pregnancy and its viability due to the production of progesterone by the corpus luteum-the main "keeper" of the normal early pregnancy. Hence, the stimulatory effect of GH on ovarian steroidogenic cell function may play a major role in endometrial function and dysfunction via its effect on ovary (see Figure 1 for the proposed mechanisms of $\mathrm{GH}$ action on endometrium).

\section{CLINICAL USE OF GH AND EFFECT ON THE ENDOMETRIUM}

Initial reports on the use of $\mathrm{GH}$ in clinical practice come from cases of hypogonadotropic hypogonadism or panhypopituitarism (46). Subsequently, the use of GH has been expanded on different patient population, such as women with poor ovarian reserve, poor responders, or with poor oocyte quality due to advanced maternal age $(25,47,48)$. In general, $\mathrm{GH}$ administration in the infertility clinic setting has focused on $\mathrm{GH}$ effects on oocyte, and the effect on endometrium has been largely overlooked.

Subsequently, the attention has been turned onto the endometrium, and interesting observations have been made suggesting positive effect of growth hormone treatment on endometrial thickness and implantation potential (see Table 1 for the studies). A case report of a patient with panhypopituitarism demonstrated improved endometrial thickness and successful implantation and pregnancy after adding growth hormone to the treatment protocol following multiple failed in vitro fertilization (IVF)/embryo transfer cycles (55). Alternatively, a study on 20 patients with documented $\mathrm{GH}$ deficiency reported improved embryo quality, but no improvement in endometrial thickness, when supplemented with GH in IVF cycle (15) (Table 1). Below we will discuss the available literature on the use of $\mathrm{GH}$ in various clinical IVF settings.

\section{Infertile Patients With Recurrent Implantation Failure}

This is a group of patients that fail to achieve pregnancy in fresh or frozen embryo transfer cycles despite appropriate endometrial development (thickness and pattern) and good quality embryo transferred. Patients with recurrent implantation failure (RIF), having undergone three or more embryo transfer cycles after IVF treatment without a clinical pregnancy, are among the most difficult patients to treat, with no proven standard treatment. Impaired endometrial maturation is suggested as a common cause for RIF (56-58), making it a target patient group who could potentially benefit from GH co-administration during IVF procedure. Chen et al. study on 42 RIF patients undergoing IVF treatment found that $\mathrm{GH}$ treatment throughout the stimulation increased the endometrial thickness and consequent pregnancy and live birth rates among young patients $<35$ years old supplemented with GH when compared to no GH RIF group (19). Patients in both groups had similar peak estradiol levels and similar number of oocytes retrieved (19). While it is unclear if the difference in endometrial thickness of $11.61 \pm 2.9$ vs. $9.7 \pm 1.46 \mathrm{~mm}$ between study and control groups, respectively was crucial in achieving higher pregnancy rates in the study group, the observation is nevertheless important. This has been reported again in the second study, a randomized clinical trial including 70 RIF patients in oocyte donation program (as an ideal model for assessing GH effect on patient's endometrium without 
A

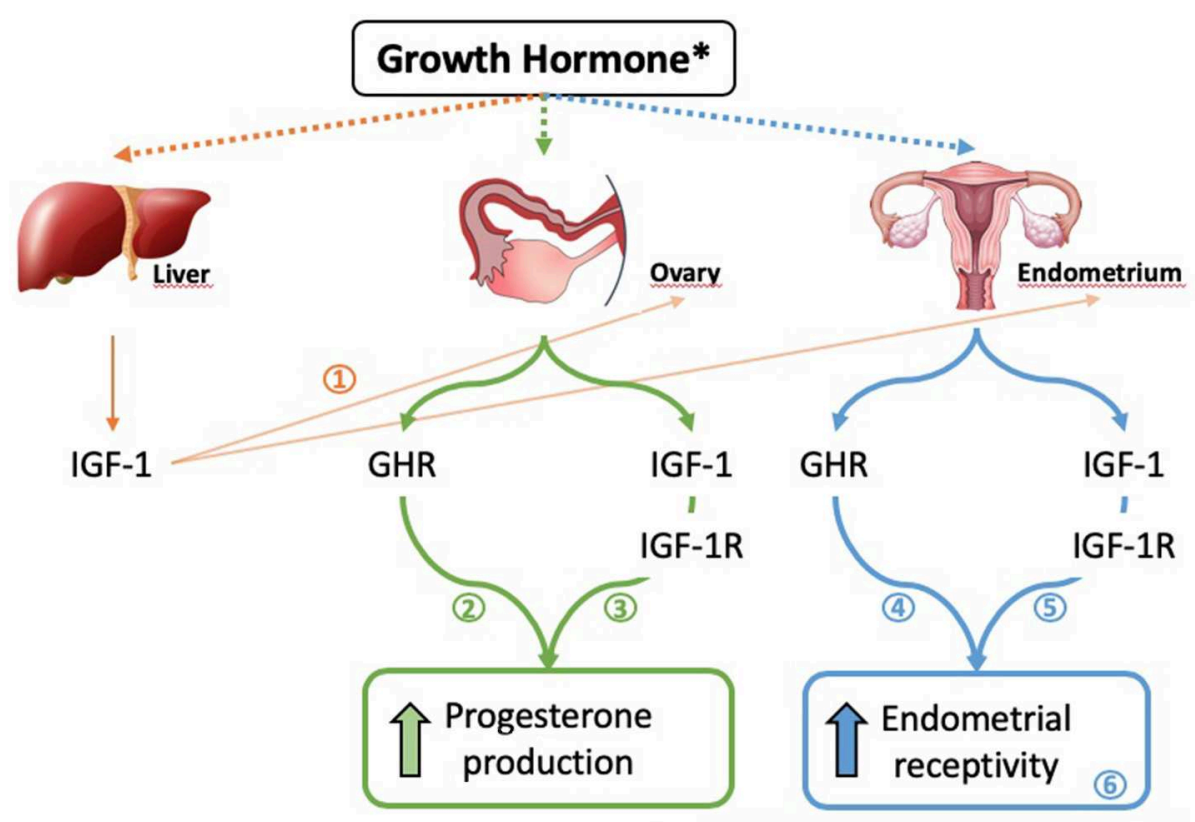

${ }^{*} \mathrm{GH}$ is produced in anterior pituitary, but can be locally produced in small amounts in reproductive tissues. GH receptor (GHR) is expressed in granulosa cells and endometrial epithelial cells

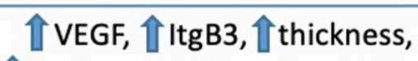
$\uparrow$ endometrial blood perfusion Higher implantation and

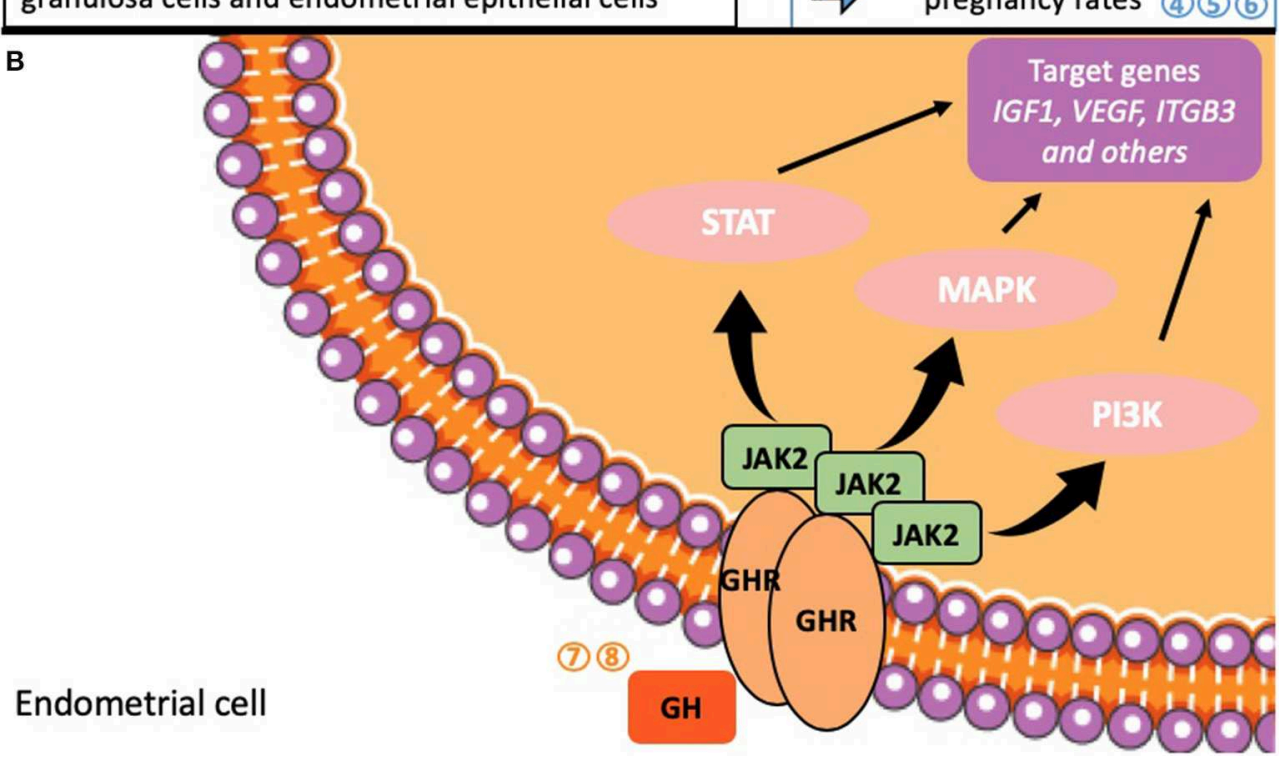

FIGURE 1 | Possible mechanisms of GH effects on ovarian and endometrial function (A) and on endometrial cells (B). Numbers in the figure indicate studies where the information is presented in detail: 1 (39); 2 (40, 41); 3 (42); 4 (23); 5 (21); 6 (43); 7 (44); 8 (45).

confounding factors of ovarian age and response) (20). In that study patients, who were treated with GH throughout medicated frozen embryo transfer cycle demonstrated significantly thicker endometrium, $9.3 \pm 1.5 \mathrm{~mm}$ vs. $8.6 \pm 1.0 \mathrm{~mm}$, respectively, and higher pregnancy and live birth rates compared with RIF patients in the placebo group (20) (Table 1).

These are the first two studies assessing GH effects on endometrium in RIF patients, and, although the findings are promising, clearly more studies on larger patient population, as well as randomized clinical trials (RCTs), are needed for any clinically meaningful conclusions. It is well-accepted that endometrial thickness does not necessarily mean that the endometrium is receptive, yet it is considered as a measure of endometrial maturity, and optimal growth of the endometrium $(>7 \mathrm{~mm})$ is required for a successful embryo implantation (59-61). 
TABLE 1 | Studies assessing the effect of growth hormone (GH) co-treatment in in vitro fertilization (fresh treatment cycles and frozen embryo transfer cycles) on endometrium

\begin{tabular}{|c|c|c|c|c|c|c|c|c|c|c|}
\hline \multirow[t]{2}{*}{ Study } & \multirow[t]{2}{*}{ RCT } & \multirow{2}{*}{$\begin{array}{l}\text { Study group; } \\
\text { Ethnicity }\end{array}$} & \multirow{2}{*}{$\begin{array}{l}\text { GH/control } \\
\text { (mean age) }\end{array}$} & \multirow[t]{2}{*}{ Inclusion criteria } & \multirow[t]{2}{*}{ Exclusion criteria } & \multirow[t]{2}{*}{ Intervention } & \multirow[t]{2}{*}{ Primary outcome } & \multicolumn{3}{|c|}{ Effect on endometrial thickness (mm) } \\
\hline & & & & & & & & GH & Control & $p$-value \\
\hline \multicolumn{11}{|c|}{ FRESH EMBRYO TRANSFER CYCLE } \\
\hline $\begin{array}{l}\text { Rajesh et al. } \\
\text { (15) }\end{array}$ & No & $\begin{array}{l}\text { Infertile women } \\
\text { with GH } \\
\text { deficiency; } \\
\text { Chinese }\end{array}$ & $\begin{array}{l}20 / 20^{*} \\
(32.9 \text { y) } \\
\text { *same women } \\
\text { cycle before } \\
\text { without GH } \\
\text { served } \\
\text { as controls }\end{array}$ & $\begin{array}{l}\text { GH deficiency based on } \\
\text { clonidine test; previous } \\
\text { IVF cycle without GH; } \\
\text { became pregnant with } \\
\text { GH treated cycle }\end{array}$ & $\begin{array}{l}\text { Panhypopituitarism; GH } \\
\text { deficient patients with } \\
\text { previous cycle treated at } \\
\text { other hospital }\end{array}$ & $\begin{array}{l}12 \text { IU GH every 3rd day, } \\
\text { starting from GnRH } \\
\text { stimulation day until hCG } \\
\text { administration }\end{array}$ & $\begin{array}{l}\text { Improved embryo } \\
\text { quality; higher } \\
\text { fertilization rate at } \\
\text { ICSI }\end{array}$ & $11.4 \pm 1.9$ & $10.3 \pm 1.5$ & 0.108 \\
\hline $\begin{array}{l}\text { Eftekhar et al. } \\
\text { (49) }\end{array}$ & Yes & $\begin{array}{l}\text { Poor responders; } \\
\text { Iranian }\end{array}$ & $\begin{array}{l}40 / 42 \\
(36.0 \pm 4.6 \\
y / 36.2 \pm 3.7 y)\end{array}$ & $\begin{array}{l}\text { previous failed IVF-ET } \\
\text { cycles with } \leq 3 \text { oocytes, } \\
\text { and } \leq 3 \text { embryos } \\
\text { obtained; and/or E2 } \\
\text { levels } \leq 500 \mathrm{pg} / \mathrm{mL} \text { on } \\
\text { hCG day }\end{array}$ & $\begin{array}{l}\mathrm{BMI} \geq 30, \mathrm{FSH}>15 \mathrm{IU} / \mathrm{L} \text {, } \\
\text { endocrine or metabolic } \\
\text { disorders, and PCOS, } \\
\text { severe endometriosis } \\
\text { and azoospermia }\end{array}$ & $\begin{array}{l}\text { GnRH antagonist } \\
\text { protocol; +treatment } \\
\text { group } 4 \mathrm{IU} / \mathrm{d} \text { GH from } \\
\text { day } 21 \text { from previous } \\
\text { cycle until hCG triggering }\end{array}$ & $\begin{array}{l}\text { Higher number of } \\
\text { retrieved oocytes and } \\
\text { obtained embryos, } \\
\text { while no effect on } \\
\text { implantation and } \\
\text { pregnancy rates }\end{array}$ & $8.5 \pm 1.0$ & $8.1 \pm 0.9$ & $0.158^{\mathrm{a}}$ \\
\hline $\begin{array}{l}\text { Bayoumi et al. } \\
\text { (50) }\end{array}$ & Yes & $\begin{array}{l}\text { Poor responders; } \\
\text { Egyptian }\end{array}$ & $\begin{array}{l}72 / 73 \\
(34.9 \pm 4.9 \\
y / 34.8 \pm 5.6 y)\end{array}$ & $\begin{array}{l}\text { ESHRE consensus } \\
\text { criteria } 2011 \text { for poor } \\
\text { responders }\end{array}$ & $\begin{array}{l}\text { FSH > } 20 \text { IU//; previous } \\
\text { ovarian surgery; infertility } \\
\text { other than poor ovarian } \\
\text { response; endocrine } \\
\text { disorder; male factor } \\
\text { infertility }\end{array}$ & $\begin{array}{l}\text { GnRH agonist (microflare) } \\
\text { protocol; +treatment } \\
\text { group } 7.5 \mathrm{IU} / \mathrm{d} \text { GH from } \\
\text { day } 6 \text { of } \mathrm{hMG} \text { stimulation } \\
\text { until day of hCG } \\
\text { triggering }\end{array}$ & $\begin{array}{l}\text { Higher number of } \\
\text { mature oocytes and } \\
\text { embryos obtained, } \\
\text { while no effect on } \\
\text { implantation and } \\
\text { pregnancy rates }\end{array}$ & $11.9 \pm 1.6$ & $11.7 \pm 1.7$ & $0.590^{\mathrm{a}}$ \\
\hline $\begin{array}{l}\text { Dakhly et al. } \\
\text { (51) }\end{array}$ & Yes & $\begin{array}{l}\text { Poor responders; } \\
\text { Egyptian }\end{array}$ & $\begin{array}{l}74 / 74 / 68 / 71^{*} \\
(36.4 \pm 5.8 \\
y / 38.1 \pm 5.0 \\
y / 36.8 \pm 6.3 \\
\text { y/36.4 } \pm 5.8 y) \\
{ }^{*} \text { Comparison of } \\
4 \text { different GH } \\
\text { protocols, no } \\
\text { control group }\end{array}$ & $\begin{array}{l}\text { ESHRE consensus } \\
\text { criteria } 2011 \text { for poor } \\
\text { responders }\end{array}$ & $\begin{array}{l}>45 \text { y; FSH > } 20 \text { IU//; } \\
\text { previous ovarian surgery; } \\
\text { other causes of infertility } \\
\text { (other than poor } \\
\text { responder); male factor } \\
\text { of infertility }\end{array}$ & $\begin{array}{l}\text { Gr1: GnRH long protocol; } \\
\text { Gr2: GnRH short } \\
\text { protocol; Gr3: GnRH } \\
\text { antagonist protocol; Gr4: } \\
\text { GnRH miniflare protocol. } \\
\text { In all groups } 7.5 \mathrm{IU} / \mathrm{d} \mathrm{GH} \\
\text { from day } 6 \text { of hMG } \\
\text { stimulation until day of } \\
\text { hCG triggering }\end{array}$ & $\begin{array}{l}\text { The long/GH (Gr1) } \\
\text { protocol was superior } \\
\text { regarding the number } \\
\text { of oocytes retrieved } \\
\text { and fertilized. No } \\
\text { significant differences } \\
\text { in pregnancy rates }\end{array}$ & $\begin{array}{r}11.5 \pm 1.6(\mathrm{Gr} 1) \\
11.4 \pm 1.6(\mathrm{Gr} 2) \\
\mathbf{1 2 . 1} \pm \mathbf{1 . 4}(\mathrm{Gr} 3) \\
\mathbf{1 1 . 1} \pm \mathbf{1 . 8}(\mathrm{Gr} 4)\end{array}$ & NA & $\begin{array}{c}\mathbf{0 . 0 0 3}^{\mathrm{a}} \\
\text { (Gr3 vs. Gr4) }\end{array}$ \\
\hline $\begin{array}{l}\text { Bassiouny } \\
\text { et al. (13) }\end{array}$ & Yes & $\begin{array}{l}\text { Poor responders; } \\
\text { Egyptian }\end{array}$ & $\begin{array}{l}68 / 73 \\
(35.8 \pm 5.6 \\
y / 35.5 \pm 6.0 y)\end{array}$ & $\begin{array}{l}\text { ESHRE consensus } \\
\text { criteria } 2011 \text { for poor } \\
\text { responders }\end{array}$ & $\begin{array}{l}\mathrm{FSH}>20 \mathrm{IU} / \text { /; previous } \\
\text { ovarian surgery; infertility } \\
\text { other than poor ovarian } \\
\text { response }\end{array}$ & $\begin{array}{l}\text { GnRH antagonist } \\
\text { protocol; +treatment } \\
\text { group } 7.5 \mathrm{IU} / \mathrm{d} \text { GH from } \\
\text { day } 6 \text { of } \mathrm{hMG} \text { stimulation } \\
\text { until day of hCG } \\
\text { triggering }\end{array}$ & $\begin{array}{l}\text { Higher number of } \\
\text { mature oocytes and } \\
\text { embryos obtained, } \\
\text { while no effect on } \\
\text { pregnancy rates }\end{array}$ & $12.1 \pm 1.3$ & $11.6 \pm 1.6$ & $0.029^{a}$ \\
\hline
\end{tabular}




\begin{tabular}{|c|c|c|c|c|c|c|c|c|c|c|}
\hline \multirow[t]{2}{*}{ Study } & \multirow[t]{2}{*}{ RCT } & \multirow{2}{*}{$\begin{array}{l}\text { Study group; } \\
\text { Ethnicity }\end{array}$} & \multirow{2}{*}{$\begin{array}{l}\text { GH/control } \\
\text { (mean age) }\end{array}$} & \multirow[t]{2}{*}{ Inclusion criteria } & \multirow[t]{2}{*}{ Exclusion criteria } & \multirow[t]{2}{*}{ Intervention } & \multirow[t]{2}{*}{ Primary outcome } & \multicolumn{3}{|c|}{ Effect on endometrial thickness (mm) } \\
\hline & & & & & & & & GH & Control & $p$-value \\
\hline Du et al. (16) & No & $\begin{array}{l}\text { Normal } \\
\text { responders; } \\
\text { Chinese }\end{array}$ & $\begin{array}{l}556 / 558 \\
(32.8 \pm 4.3 \\
\text { y/31.6 } \pm 4.4 \mathrm{y}) \\
\left({ }^{*} \text { older women }\right. \\
\geq \mathbf{3 5} \mathbf{y : ~} \mathbf{2 7 8 / 2 6 5} \\
{ }^{* *} \text { younger } \\
\text { women }<35 \\
\text { y: } 278 / 293)\end{array}$ & $\begin{array}{l}20-45 \text { y; fallopian tube } \\
\text { malfunction or male } \\
\text { sterility; normal hormone } \\
\text { levels; normal uterine } \\
\text { cavity; regular menstrual } \\
\text { cycles, BMl < } 25\end{array}$ & $\begin{array}{l}\text { Recurrent spontaneous } \\
\text { abortion; severe pelvic } \\
\text { adhesions or } \\
\text { hydrosalpinx; } \\
\text { cerebrovascular, liver or } \\
\text { kidney disease; } \\
\text { endocrine diseases; } \\
\text { PCOS; endometriosis; } \\
\text { uterine leiomyoma; } \\
\text { adenomyosis }\end{array}$ & $\begin{array}{l}\text { Long GnRH agonist } \\
\text { protocol; +treatment } \\
\text { group } 4.5 \mathrm{IU} / \mathrm{d} \text { GH for } 5 \\
\text { days starting from day of } \\
\text { FSH administration }\end{array}$ & $\begin{array}{l}\text { Higher implantation } \\
\text { and clinical } \\
\text { pregnancy rates }\end{array}$ & $\begin{array}{r}12.2 \pm 4.7 \\
{ }^{*} \mathbf{1 2 . 0} \pm \mathbf{2 . 2} \\
{ }^{* *} 12.5 \pm 7.0\end{array}$ & $\begin{array}{l}11.8 \pm 4.8 \\
{ }^{*} 11.6 \pm 2.5 \\
{ }^{\star *} 12.0 \pm 6.8\end{array}$ & $\begin{array}{l}0.18^{\mathrm{b}} \\
{ }^{*} 0.038^{\mathrm{b}} \\
{ }^{*} 0.50^{\mathrm{b}}\end{array}$ \\
\hline $\begin{array}{l}\text { Choe et al. } \\
\text { (52) }\end{array}$ & Yes & $\begin{array}{l}\text { Infertile women } \\
\text { with diminished } \\
\text { ovarian reserve; } \\
\text { Korean }\end{array}$ & $\begin{array}{l}62 / 65 \\
(39.8 \pm 3.6 \\
y / 39.4 \pm 4.1 y)\end{array}$ & $\begin{array}{l}\geq 40 \text { y or any other factor } \\
\text { for poor ovarian } \\
\text { response; } \leq 3 \text { oocytes } \\
\text { with conventional } \\
\text { stimulation protocol; } \\
\text { antral follicle count }<5-7 \\
\text { or AMH }<0.5-1.1 \mathrm{ng} / \mathrm{ml} \text {; } \\
\text { normal uterus; regular } \\
\text { menstrual cycle }\end{array}$ & $\begin{array}{l}\text { Genetic cause for } \\
\text { infertility; BMI > } 30 \text {; } \\
\text { abnormal uterine } \\
\text { bleeding; ovarian tumor; } \\
\text { breast cancer; } \\
\text { hydrosalpinx; } \\
\text { contraindication for GH } \\
\text { treatment }\end{array}$ & $\begin{array}{l}\text { GnRH antagonist } \\
\text { protocol; +treatment } \\
\text { group sustained-release } \\
\text { GH ( } 20 \mathrm{mg} \text { ) } 3 \times \text { before } \\
\text { and during COS } \\
\text { (mid-luteal, late luteal, } \\
\text { cycle day 2) }\end{array}$ & $\begin{array}{l}\text { Higher number of } \\
\text { mature oocytes } \\
\text { obtained, while no } \\
\text { effect on pregnancy } \\
\text { rates }\end{array}$ & $8.8 \pm 2.2$ & $9.1 \pm 1.9$ & $0.24^{a}$ \\
\hline $\begin{array}{l}\text { Dakhly et al. } \\
\text { (53) }\end{array}$ & Yes & $\begin{array}{l}\text { Poor responders; } \\
\text { Egyptian }\end{array}$ & $\begin{array}{l}120 / 120 \\
(36.4 \pm 4.4 \\
y / 36.2 \pm 4.5 y)\end{array}$ & $\begin{array}{l}\text { ESHRE consensus } \\
\text { criteria } 2011 \text { for poor } \\
\text { responders }\end{array}$ & $\begin{array}{l}>45 \text { y; FSH > } 20 \text { IU/l; } \\
\text { previous ovarian surgery; } \\
\text { other causes of infertility } \\
\text { (other than poor } \\
\text { responder); male factor } \\
\text { of infertility }\end{array}$ & $\begin{array}{l}\text { GnRH long protocol; } \\
\text { +treatment group } 7.5 \\
\text { IU/d GH from day } 21 \text { of } \\
\text { previous cycle until day } \\
\text { of hCG triggering }\end{array}$ & $\begin{array}{l}\text { Higher number of } \\
\text { oocytes and embryos } \\
\text { obtained, while no } \\
\text { effect on implantation } \\
\text { and pregnancy rates }\end{array}$ & $11.8 \pm 1.3$ & $11.3 \pm 1.2$ & $<0.001^{a}$ \\
\hline $\begin{array}{l}\text { Chen et al. } \\
\text { (19) }\end{array}$ & No & $\begin{array}{l}\text { Recurrent } \\
\text { implantation failure } \\
\text { (RIF) patients; } \\
\text { Chinese }\end{array}$ & $\begin{array}{l}22 / 20 \\
(33.9 \pm 2.9 \\
y / 34.0 \pm 3.4 y)\end{array}$ & $\begin{array}{l}\text { Normal hormone levels; } \\
\text { no use of synthetic } \\
\text { hormones }>3 \text { months } \\
\text { prior to entry }\end{array}$ & $\begin{array}{l}\text { Prior endometrial } \\
\text { resection or endometrial } \\
\text { polyps; antiphospholipid } \\
\text { syndrome; infectious } \\
\text { disease; hyperthyroidism; } \\
\text { hyperprolactinemia; } \\
\text { chromosomal } \\
\text { abnormalities; } \\
\text { thalassemia; male factors }\end{array}$ & $\begin{array}{l}\text { GnRH; +treatment group } \\
4 \mathrm{IU} / \mathrm{d} \text { GH through } \\
\text { stimulation until the day } \\
\text { of hCG administration }\end{array}$ & $\begin{array}{l}\text { Higher clinical } \\
\text { pregnancy and live } \\
\text { birth rates }\end{array}$ & $11.6 \pm 2.9$ & $9.7 \pm 1.5$ & $0.009^{a}$ \\
\hline Liu et al. (24) & No & $\begin{array}{l}\text { Normal } \\
\text { responders; } \\
\text { Chinese }\end{array}$ & $\begin{array}{l}781 / 781(31.3 \pm \\
3.6 y / 31.3 \pm 3.3 \\
\text { y) }\end{array}$ & $\begin{array}{l}\text { Normal ovarian response; } \\
\text { age } 20-40 \text { y; poor quality } \\
\text { embryos in previous } \\
\text { IVF/ICSI; repetitive fresh } \\
\text { or frozen ET without } \\
\text { pregnancy }\end{array}$ & $\begin{array}{l}\text { Poor or high ovarian } \\
\text { response; adjuvant } \\
\text { therapy as DHEA, } \\
\text { CoQ10; serious and } \\
\text { unstable diseases } \\
\text { (cardiovascular, } \\
\text { cerebrovascular } \\
\text { diseases); recurrent } \\
\text { spontaneous abortion; } \\
\text { male factor infertility }\end{array}$ & $\begin{array}{l}\text { GH treatment group } 2 \\
\text { IU/4 IU GH daily since } \\
\text { day } 2 \text { of previous cycle (6 } \\
\text { weeks GH pretreatment) } \\
\text { or day } 2 \text { from ovarian } \\
\text { stimulation until hCG } \\
\text { trigger ( } 2 \text { weeks GH } \\
\text { pretreatment) }\end{array}$ & $\begin{array}{l}\text { Increased pregnancy } \\
\text { rate }\end{array}$ & $12.0 \pm 2.2$ & $11.6 \pm 2.8$ & $0.036^{a}$ \\
\hline
\end{tabular}




\begin{tabular}{|c|c|c|c|c|c|c|c|c|c|c|}
\hline \multirow[t]{2}{*}{ Study } & \multirow[t]{2}{*}{$\mathrm{RCT}$} & \multirow{2}{*}{$\begin{array}{l}\text { Study group; } \\
\text { Ethnicity }\end{array}$} & \multirow{2}{*}{$\begin{array}{l}\mathrm{GH} / \text { control } \\
\text { (mean age) }\end{array}$} & \multirow[t]{2}{*}{ Inclusion criteria } & \multirow[t]{2}{*}{ Exclusion criteria } & \multirow[t]{2}{*}{ Intervention } & \multirow[t]{2}{*}{ Primary outcome } & \multicolumn{3}{|c|}{ Effect on endometrial thickness (mm) } \\
\hline & & & & & & & & GH & Control & $p$-value \\
\hline \multicolumn{11}{|c|}{ FROZEN EMBRYO TRANSFER/OOCYTE DONATION PROTOCOL } \\
\hline Wu et al. (43) & NA & $\begin{array}{l}\text { Patients with thin } \\
\text { endometrium; } \\
\text { Chinese }\end{array}$ & $\begin{array}{l}32 / 30 \\
\text { (NA) }\end{array}$ & NA & NA & $\begin{array}{l}\text { HRT; +treatment group } \\
\text { subcutaneous injections } \\
\text { of GH }\end{array}$ & $\begin{array}{l}\text { Improved endometrial } \\
\text { blood flow and } \\
\text { increased } \\
\text { endometrial thickness }\end{array}$ & $8.8 \pm 1.3$ & $7.1 \pm 1.9$ & $<0.05$ \\
\hline Yu et al. (54) & No & $\begin{array}{l}\text { Patients with } \\
\text { persistent thin } \\
\text { endometrium; } \\
\text { Chinese }\end{array}$ & $\begin{array}{l}5 / 5^{*} \\
(32.2 \pm 5.5 \mathrm{y}) \\
{ }^{*} \text { same women } \\
\text { served as } \\
\text { controls before } \\
\text { entering } \\
\text { GH treatment }\end{array}$ & $\begin{array}{l}\text { Regular menstrual cycle; } \\
\text { use of artificial cycle; } \\
\text { endometrium } \geq 7 \mathrm{~mm} \text {; no } \\
\text { abnormalities with } \\
\text { hysteroscopy; }<40 \mathrm{y} \text {; } \\
\text { pelvic tubal or male } \\
\text { factor infertility }\end{array}$ & NA & $\begin{array}{l}\mathrm{HRT} \text {; }+\mathrm{GH} \text { treatment } \\
\text { with } 4-5 \text { intrauterine } \mathrm{GH} \\
\text { perfusions of } 6 \mathrm{IU} \mathrm{GH} \\
\text { diluted with } 0.5 \mathrm{ml} 0.9 \% \\
\text { saline on } 9 \text { th to } 12 \text { th day } \\
\text { of the cycle (bed rest } \\
15 \mathrm{~min} \text { ) }\end{array}$ & $\begin{array}{l}\text { Improved endometrial } \\
\text { thickness and } \\
\text { receptivity }\end{array}$ & $8.0 \pm 0.6$ & $5.8 \pm 0.7$ & $<0.05^{\mathrm{b}}$ \\
\hline $\begin{array}{l}\text { Xue-Mei et al. } \\
\text { (23) }\end{array}$ & No & $\begin{array}{l}\text { Infertile women } \\
\text { undergoing FET; } \\
\text { Chinese }\end{array}$ & $\begin{array}{l}77 \text { Gr1/ } 77 \text { Gr2/ } \\
76 \text { controls } \\
\text { (cycles; } n=240 \\
\text { women) } \\
(30.3 \pm 4.1 \\
\text { y/31.3 } \pm 5.0 \\
y / 30.7 \pm 4.3 \mathrm{y} \text { ) }\end{array}$ & $\begin{array}{l}\leq 38 \text { y; vitrified embryos } \\
\text { not older than } 2 \mathrm{y} ; \geq 2 \\
\text { embryos frozen }\end{array}$ & $\begin{array}{l}\text { Congenital or acquired } \\
\text { uterine malformation; } \\
\text { endometrial polyps; } \\
\text { submucosal fibroids; } \\
\text { intrauterine adhesion; } \\
\text { severe endometriosis or } \\
\text { adenomyosis; diabetes } \\
\text { mellitus; abnormal blood } \\
\text { clotting }\end{array}$ & $\begin{array}{l}\text { HRT with oral estradiol } \\
\text { valerate from cycle day } 3 . \\
\text { +treatment group } 1 \\
\text { (Gr1): } 4 \mathrm{IU} / \mathrm{d} \mathrm{GH} \\
\text { injections from cycle day } \\
8 \text { until prog injection; } \\
\text { +treatment group } 2 \\
\text { (Gr2): } 4 \mathrm{IU} / \mathrm{d} \text { GH } \\
\text { injections from cycle day } \\
3 \text { until prog injection }\end{array}$ & $\begin{array}{l}\text { Higher implantation, } \\
\text { clinical pregnancy } \\
\text { and live birth rates }\end{array}$ & $\begin{array}{l}9.2 \pm 0.9(\mathrm{Gr} 1) \\
\mathbf{9 . 6} \pm \mathbf{1 . 0}(\mathrm{Gr} 2)\end{array}$ & $9.2 \pm 0.8$ & $<0.001^{\mathrm{b}}$ \\
\hline $\begin{array}{l}\text { Altmäe et al. } \\
(20)\end{array}$ & Yes & $\begin{array}{l}\text { RIF patients with } \\
\text { fresh donated } \\
\text { oocytes; Spanish }\end{array}$ & $\begin{array}{l}35 / 70 \\
(42.2 \pm 4.5 \\
\text { y/42.4 } \pm 3.7 \\
\text { y/43.8 } \pm 2.5 \text { y) } \\
\text { (35 GH RIF; } \\
\text { Control Gr1 } 35 \\
\text { nonGH RIF; } \\
\text { Control Gr2 } 35 \\
\text { pos controls } \\
\text { undergoing 1st } \\
\text { oocyte donation) }\end{array}$ & $\begin{array}{l}\mathrm{RIF}(\geq 2 \text { implantation } \\
\text { failures); 30-51 y }\end{array}$ & NA & $\begin{array}{l}\text { GnRH agonist + oral } \\
\text { estradiol; +treatment } \\
\text { group daily injections of } \\
1 \mathrm{mg} \mathrm{GH}(\sim 3 \mathrm{IU}) \text { for } 10 \\
\text { days of proliferative } \\
\text { phase induced by } \\
\text { exogenous oral estradiol. } \\
1-2 \text { days later vaginal } \mathrm{P} \\
\text { treatment was started }\end{array}$ & $\begin{array}{l}\text { Higher implantation, } \\
\text { pregnancy and live } \\
\text { birth rates }\end{array}$ & $9.3 \pm 1.5$ & $\begin{array}{c}8.6 \pm 1.0 \\
(\mathrm{Gr} 1 \text { non-GH)} ; \\
9.4 \pm 1.7 \\
(\mathrm{Gr} 2 \\
\text { pos control) }\end{array}$ & $0.046^{\mathrm{b}}$ \\
\hline $\begin{array}{l}\text { Yang et al. } \\
\text { (22) }\end{array}$ & No & $\begin{array}{l}\text { Patients with thin } \\
\text { endometrium; } \\
\text { Chinese }\end{array}$ & $\begin{array}{l}\text { 184/61 (cycles; } \\
n=225 \text { women) } \\
(33.7 \pm 3.6 \\
y / 33.7 \pm 3.4 y)\end{array}$ & $\begin{array}{l}<40 \text { y; receiving } 2 \\
\text { blastocysts; endometrial } \\
\text { thickness }<8 \mathrm{~mm} \text { on } \\
\text { prog administration day. } \\
\text { All patients with } \\
\text { hysteroscopy for } \\
\text { adhesions before FET }\end{array}$ & $\begin{array}{l}\text { Uterine malformations; } \\
\text { severe endometriosis or } \\
\text { adenomyosis; tumor; } \\
\text { diabetes mellitus; } \\
\text { immune abnormalities }\end{array}$ & $\begin{array}{l}\text { GnRH agonist + estradiol } \\
\text { valerate from day } 2-3 \text { of } \\
\text { cycle+ vaginal estradiol } \\
\text { after menstruation + } \\
\text { prog for } 5 \text { days; + } \\
\text { treatment group } 4.5 \mathrm{IU} \\
\text { GH every alternate day } \\
\text { subcutaneously injected } \\
\text { from day of prog } \\
\text { administration until ET }\end{array}$ & $\begin{array}{l}\text { Higher clinical } \\
\text { pregnancy and } \\
\text { implantation rates }\end{array}$ & $6.6 \pm 2.9$ & $6.7 \pm 0.7$ & $0.24^{c}$ \\
\hline
\end{tabular}




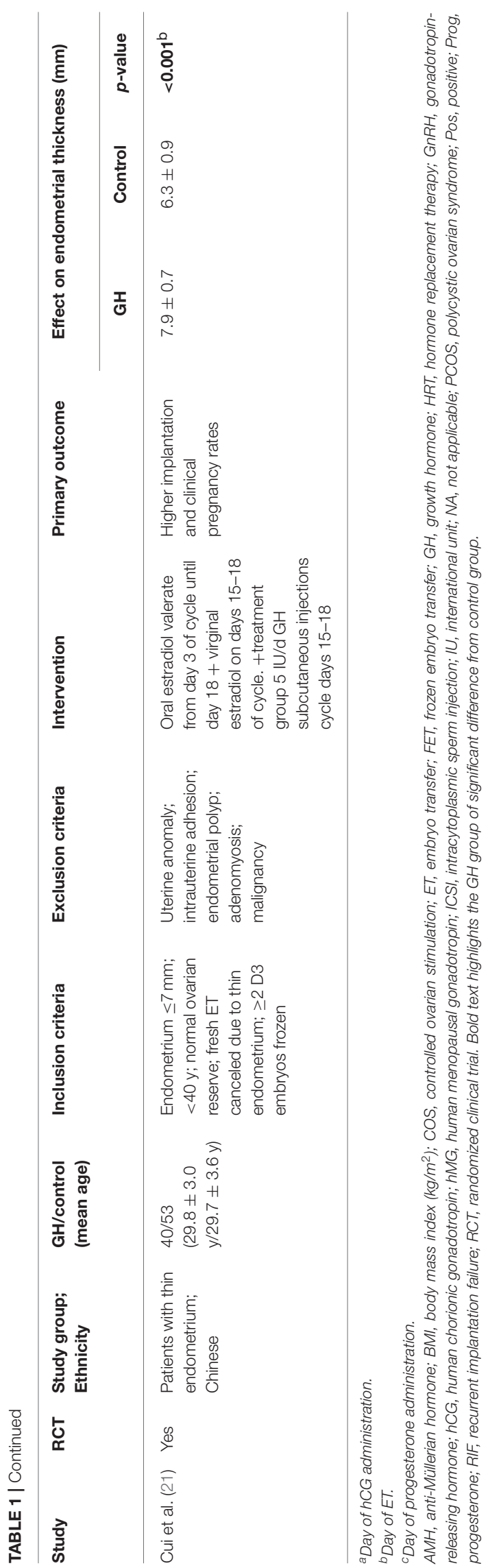

\section{Thin Endometrium}

Infertile women with thin endometrium represent another potential patient population that could benefit from the GH administration. All studies on GH co-treatment during treatment of infertile women with thin endometrium were conducted in frozen embryo transfer (FET) cycles, where GH was administered during the endometrial preparation for FET $(21,22,43,54)$ (Table 1). The largest study by Yang et al. was conducted on 225 infertile women, and did not detect any significant GH effect on endometrial thickness, while reporting significantly higher clinical pregnancy and implantation rates (22). They assessed $\mathrm{GH}$ effect on endometrial thickness on the day of progesterone administration, which could explain the difference in their results from the rest of the studies. The other three studies all noted significant improvement in endometrial thickness on the day of embryo transfer among patients with thin endometrium after administering GH throughout the FET cycle $(21,43,54)$, and significantly higher implantation and clinical pregnancy rates (21). Wu et al. study also detected improved endometrial blood flow in the GH-administered patient group (43), similar to later findings by Xue-Mei et al. study (23), who showed increased VEGF expression and improved perfusion of the uterine arteries in the group of infertile women treated with GH. In line with above, Cui et al. study detected VEGF up-regulation together with ITGB3 and IGF-1 in endometrial cells when exposed to GH (21). The state of high blood flow resistance and VEGF downregulation with inadequate epithelial growth and vascularization have been described as pathophysiologic characteristics of thin endometrium (62), and subendometrial blood flow on the day of embryo transfer is related to the implantation and pregnancy rate in IVF (63). Cui et al. concluded that up-regulated VEGF in their study setting, in the GH group, partly resulted in the increase of subendometrial blood flow and thereby improved endometrial receptivity (21). Nevertheless, the exact mechanisms of GH actions on the endometrium and endometrial receptivity in general are to be unraveled in future studies. Also new studies with larger study groups and well-designed RCTs are required in order to clarify whether infertile women with thin endometrium benefit from the GH treatment.

\section{Poor Responders}

Women with poor ovarian response in ART is another patient group where $\mathrm{GH}$ co-treatment in stimulation protocols have been studied. All these studies (see Table 1) have been RCTs, however with limited sample sizes, and all have reported beneficial effect of GH administration on the number and quality of oocytes and on the number of embryos obtained. Remarkably, while some improvement of endometrial thickness has been noted, those studies failed to show any beneficial effect on clinical pregnancy and live birth rates $(13,49-53)$. Based on these findings, one could conclude that GH co-treatment in poor responders with normal endometrium does not seem to have any significant impact on endometrial receptivity and hence pregnancy rates. Nevertheless, we should be cautious in drawing preliminary and potentially wrong conclusions in this type of studies without taking into careful consideration all potential confounders, including quality and number of embryos transferred, cleavage 
vs. blastocyst stage embryos and even type of luteal support provided in fresh and/or frozen embryo transfer cycles (64). In addition, the total productivity rate from a single oocyte retrieval is highest when more and better quality embryos are obtained, which can be exactly the case with GH-supplemented cycles in poor responders, resulting in higher cumulative pregnancy rates rather than per cycle success in this group of patients. Clearly, carefully designed large studies with transfers of single good quality embryo (fresh and frozen) are warranted, albeit quite challenging to perform, in order to clarify whether endometrial receptivity in infertile women with poor response in ART would benefit from GH administration.

\section{Normal Responders}

Thus far, the largest group of infertile patients involved in studies on GH administration during IVF has been the normal responders (Table 1). The first study was performed on 240 infertile women undergoing FET, where two different GH supplementation protocols were compared-GH administration throughout the FET, and a single GH injection on day 8 of estrogen treatment (23). Notably, significant endometrial thickness improvement together with higher embryo implantation, clinical pregnancy, and live birth rates were detected among women with longer GH administration (23). The authors also noted that the longer GH addition to the treatment protocol increased the levels of estradiol, IGF-1, and VEGF serum levels, and improved perfusion of the uterine endometrial arcuate artery (23). The pulsatility index, resistance index, and peak systolic velocity/end diastolic velocity of the uterine arcuate arteries represent the resistance of blood flow from the point of measurement downstream; increased impedance of these arteries might correlate with poor endometrial receptivity and clinical outcomes (65).

The next studies analyzed 1,114 (16) and 1,562 (24) infertile women, respectively undergoing ovarian stimulation for IVF with GH co-administration throughout the stimulation, and a positive $\mathrm{GH}$ effect on endometrial thickness in addition to the higher clinical pregnancy rates was detected in study compared to control groups. GH effect on endometrial thickness was significantly increased among older infertile women of $\geq 35$ years old compared to $<35$ years old, while both groups exhibited higher implantation and clinical pregnancy rates, most likely attributed to the higher number of high quality embryos obtained in GH-treated groups (16). In humans, changes in GH secretion could be age-related, as post-adolescence the secretion of GH decreases with age, which is why GH hyposecretion is observed in older patients (66). GH insufficiency can disrupt ovarian function and lead to reproductive difficulties (66). As mentioned above, in Du et al. study (16), GH-treated older women ( $\geq 35$ years old) had implantation and clinical pregnancy rates more than two times higher than those observed during IVF cycles without $\mathrm{GH}$. This result suggested that adding GH might be beneficial for older patients.

To conclude, research on the effects of GH co-treatment in ART among normal responders has been performed on sufficiently powered studies in terms of the sample size, nevertheless as all these studies were not randomized controlled trials, further well-designed research is needed to objectively assess the GH effect on ART outcomes in (young) women with normal ovarian reserve and normal response to ovarian stimulation.

\section{Future Perspectives}

Further studies are warranted in order to determine the optimal dose, time, and duration of GH administration and to investigate the long-term safety of GH for patients and their offspring. The dosage and treatment duration of GH differed among conducted studies (see Table 1). Because of the limited experience with the $\mathrm{GH}$ co-treatment protocols, there is a lack of evidence to support the superiority of one over the other. In all the protocols used (see Table 1), GH was administered via subcutaneous injections, except for one study where GH intrauterine perfusion in 5 patients with non-responsive thin endometrium was successfully used (54).

Another crucial part is to define the appropriate patient population that would truly benefit from GH treatment for improving their uterine lining quality in terms of thickness and/or receptivity. GH seems to promote endometrial growth, and its use could be considered in women whose endometrium does not grow and/or mature sufficiently with standard treatment protocols. In addition, the current review concludes that even normal responders could potentially benefit from the GH administration in IVF programs, however, the improved pregnancy rates in some of the studies utilizing fresh IVF cycles could not be separated from improved embryo quality. While endometrial thickness and pattern upon GH administration has been recorded and reported, evaluation of endometrial receptivity is not as simple. Future studies need to focus on the molecular level in order to evaluate the endometrial transcriptome/proteome/secretome (67), with emphasis on receptivity markers to understand and clarify the possible mechanisms of GH on endometrial receptivity. An ideal setting would be to design an RCT with GH-supplemented mock cycles vs. control, during which endometrial receptivity could be studied on molecular level in detail (transcriptomics and/or use of commercially available endometrial receptivity tests; epigenomics and/or proteomics analyses). The mock cycle could be followed by a "true" FET cycle to enable evaluation and correlation to pregnancy rates. To sum up, undoubtedly more research on larger cohorts with carefully designed studies [as highlighted in a recent comment (64)] is needed to identify the patient group in whom the addition of GH to the treatment protocol in IVF programs will be most valuable.

Sample size and objectively designed studies (randomized clinical trials) is a delicate topic in ART as strict doubleblind, placebo-controlled, RCTs are difficult to accomplish (68). It is extremely hard to perform fully blinded RCTs in IVF because of the patient recruitment issues, where aging women prefer not to participate in the placebo group that requires commitment for several months of their reproductive lifespan and which ultimately may not help them achieve pregnancy (68). Understandingly, patients tend to opt for any additional treatment, cost permitting, that would potentially help them to become pregnant. As a result, the studies of $\mathrm{GH}$ treatment effects 
on IVF outcomes are rather limited on its sample size and/or are retrospective or observational in nature; nonetheless, they provide important data concerning therapeutic interventions in IVF and open up future possibilities for improving infertility treatment protocols.

\section{CONCLUSIONS}

The current review summarizes the recent data on GH co-treatment effects on endometrial parameters in assisted reproduction and proposes possible mechanisms of GH actions in the endometrium. Studies are indicating that co-treatment with GH could improve the endometrial thickness, and possibly receptivity among infertile women. This effect might occur through increasing endometrial blood perfusion and the expression of genes and proteins related to endometrial receptivity such as VEGF and ITGB3 together with IGF-1, however the exact mechanisms in the endometrium remain to be clarified.

Whether GH administration during IVF is useful and which patient groups could benefit from it needs further investigation, but the preliminary data suggest that women suffering RIF, patients with thin endometrium and older normo-responders could benefit from GH treatment when undergoing ART. Still, carefully designed and sufficiently powered cohort studies, RCTs, are required in the field in order to establish the most suitable therapeutic regimen for these patients and to clarify

\section{REFERENCES}

1. Wilcox AJ, Baird DD, Weinberg CR. Time of implantation of the conceptus and loss of pregnancy. N Engl J Med. (1999) 340:1796-9. doi: 10.1056/NEJM199906103402304

2. Carson DD, Lagow E, Thathiah A, Al-Shami R, Farach-Carson MC, Vernon $\mathrm{M}$, et al. Changes in gene expression during the early to midluteal (receptive phase) transition in human endometrium detected by high-density microarray screening. Mol Hum Reprod. (2002) 8:871-9. doi: 10.1093/molehr/8.9.871

3. Altmäe S, Reimand JR, Hovatta O, Zhang P, Kere J, Laisk T, et al. Research resource: interactome of human embryo implantation: identification of gene expression pathways, regulation, and integrated regulatory networks. Mol Endocrinol. (2012) 26:203-17. doi: 10.1210/me.2011-1196

4. Norwitz ER, Schust DJ, Fisher SJ. Implantation and the survival of early pregnancy. N Engl J Med. (2001) 345:1400-8. doi: 10.1056/NEJMra000763

5. Edwards RG. Clinical approaches to increasing uterine receptivity during human implantation. Hum Reprod. (1995) 10(Suppl. 2):60-6. doi: 10.1093/humrep/10.suppl_2.60

6. Margalioth EJ, Ben-Chetrit A, Gal M, Eldar-Geva T. Investigation and treatment of repeated implantation failure following IVF-ET. Hum Reprod. (2006) 21:3036-43. doi: 10.1093/humrep/del305

7. Hviid MM, Macklon N. Immune modulation treatmentswhere is the evidence? Fertil Steril. (2017) 107:1284-93. doi: 10.1016/j.fertnstert.2017.04.009

8. Evers JLHH. Is RIF rife? Hum Reprod. (2016) 31:2661. doi: $10.1093 /$ humrep/dew277

9. Hull KL, Harvey S. Growth hormone and reproduction: a review of endocrine and autocrine/paracrine interactions. Int J Endocrinol. (2014) 2014:234014. doi: $10.1155 / 2014 / 234014$

10. Cailleau J, Vermeire S, Verhoeven G. Independent control of the production of insulin-like growth factor I and its binding protein the confusion arisen from various studies that have shown either inconsistent or conflicting findings, used small patient cohorts and/or have been poorly designed with no blinding or placebo controls.

\section{AUTHOR CONTRIBUTIONS}

SA and LA equally contributed to the review idea and manuscript writing.

\section{FUNDING}

This study was supported by the University of Granada Plan Propio de Investigación 2016-Excellence actions: Unit of Excellence on Exercise and Health (UCEES)-and Plan Propio de Investigación 2018-Programa Contratos-Puente, and the Junta de Andalucía, Consejería de Conocimiento, Investigación y Universidades, European Regional Development Funds (ref. SOMM17/6107/UGR); and the Spanish Ministry of Economy, Industry and Competitiveness (MINECO), and European Regional Development Fund (FEDER): grants RYC-2016-21199 and ENDORE SAF2017-87526.

\section{ACKNOWLEDGMENTS}

We thank Dr. Alberto Sola-Leyva for his help in the figure preparation.

by cultured testicular cells. Mol Cell Endocrinol. (1990) 69:79-89. doi: 10.1016/0303-7207(90)90091-L

11. Kolibianakis EM, Venetis CA, Diedrich K, Tarlatzis BC, Griesinger G. Addition of growth hormone to gonadotrophins in ovarian stimulation of poor responders treated by in-vitro fertilization: a systematic review and meta-analysis. Hum Reprod Update. (2009) 15:613-22. doi: 10.1093/humupd/dmp026

12. Li X-LL, Wang L-PP, Lv F, Huang X-MM, Wang L-PP, Pan Y, et al. The influence of different growth hormone addition protocols to poor ovarian responders on clinical outcomes in controlled ovary stimulation cycles: a systematic review and meta-analysis. Medicine. (2017) 96:e6443. doi: 10.1097/MD.0000000000006443

13. Bassiouny YA, Dakhly DMR, Bayoumi YA, Hashish NM. Does the addition of growth hormone to the in vitro fertilization/intracytoplasmic sperm injection antagonist protocol improve outcomes in poor responders? A randomized, controlled trial. Fertil Steril. (2016) 105:697-702. doi: $10.1016 /$ j.fertnstert.2015.11.026

14. Kucuk T, Kozinoglu H, Kaba A. Growth hormone co-treatment within a GnRH agonist long protocol in patients with poor ovarian response: a prospective, randomized, clinical trial. J Assist Reprod Genet. (2008) 25:123-7. doi: 10.1007/s10815-008-9212-7

15. Rajesh H, Yong YY, Zhu M, Chia D, Yu SL. Growth hormone deficiency and supplementation at in-vitro fertilisation. Singapore Med J. (2007) 48:514-518.

16. Du FX, Yang XH, Li J, Hao M, Goa YH. Growth hormone co-treatment within a GnRH agonist long protocol improves implantation and pregnancy rates in patients undergoing IVF-ET. Arch Gynecol Obstet. (2016) 294:877-83. doi: $10.1007 /$ s00404-016-4163-1

17. Hazout A, Junca Am, Ménézo Y, Demouzon J, Cohen-Bacrie P. Effect of growth hormone on oocyte competence in patients with multiple IVF failures. Reprod Biomed Online. (2009) 18:664-70. doi: 10.1016/S1472-6483(10)60011-9 
18. Lattes K, Brassesco M, Gomez M, Checa MA. Low-dose growth hormone supplementation increases clinical pregnancy rate in poor responders undergoing in vitro fertilisation. Gynecol Endocrinol. (2015) 31:565-8. doi: 10.3109/09513590.2015.1025378

19. Chen Y, Liu F, Nong Y, Ruan J, Guo Q, Luo M, et al. Clinical efficacy and mechanism of growth hormone action in patients experiencing repeat implantation failure. Can J Physiol Pharmacol. (2018) 96:929-32. doi: 10.1139/cjpp-2017-0786

20. Altmäe S, Mendoza-Tesarik R, Mendoza C, Mendoza N, Cucinelli F, Tesarik J. Effect of growth hormone on uterine receptivity in women with repeated implantation failure in an oocyte donation program: a randomized controlled trial. J Endocr Soc. (2018) 2:96-105. doi: 10.1210/js.2017-00359

21. Cui N, Li AM, Luo ZY, Zhao ZM, Xu YM, Zhang J, et al. Effects of growth hormone on pregnancy rates of patients with thin endometrium. J Endocrinol Invest. (2019) 42:27-35. doi: 10.1007/s40618-018-0877-1

22. Yang J-Y, $\mathrm{Li} \mathrm{H}, \mathrm{Lu} \mathrm{N}$, Li L, Sun X-X. Influence of growth hormone supplementation in patients with thin endometrium undergoing frozen embryo transfer. Reprod Dev Med. (2019) 3:49-53. doi: 10.4103/2096-2924.255983

23. Xue-Mei W, Hong J, Wen-Xiang Z, Yang L. The effects of growth hormone on clinical outcomes after frozen - thawed embryo transfer. Int J Gynecol Obstet. (2016) 133:347-50. doi: 10.1016/j.ijgo.2015.10.020

24. Liu X, Bai H, Xie J, Shi J. Growth hormone co-treatment on controlled ovarian stimulation in normal ovarian response women can improve embryo quality. Gynecol Endocrinol. (2019) 35:787-91. doi: 10.1080/09513590.2019.1590545

25. Tesarik J, Hazout A, Mendoza C. Improvement of delivery and live birth rates after ICSI in women aged $>40$ years by ovarian co-stimulation with growth hormone. Hum Reprod. (2005) 20:2536-41. doi: 10.1093/humrep/dei066

26. Yovich JL, Stanger JD. Growth hormone supplementation improves implantation and pregnancy productivity rates for poor-prognosis patients undertaking IVF. Reprod Biomed Online. (2010) 21:37-49. doi: 10.1016/j.rbmo.2010.03.013

27. Duffy JM, Ahmad G, Mohiyiddeen L, Nardo LG, Watson A. Growth hormone for in vitro fertilization. Cochrane Database Syst Rev. (2010) 7: CD000099. doi: 10.1002/14651858.CD000099.pub3

28. Sbracia M, Scarpellini F, Poverini R, Alò PL, Rossi G, Di Tondo U, et al. Immunohistochemical localization of the growth hormone in human endometrium and decidua. Am J Reprod Immunol. (2004) 51:112-6. doi: 10.1046/j.8755-8920.2003.00127.x

29. Shifren JL, Osathanondh R, Yeh J. Human fetal ovaries and uteri: developmental expression of genes encoding the insulin, insulin-like growth factor I, and insulin-like growth factor II receptors. Fertil Steril. (1993) 59:1036-40. doi: 10.1016/S0015-0282(16)55924-X

30. Thiet MP, Osathanondh R, Yeh J. Localization and timing of appearance of insulin, insulin-like growth factor-I, and their receptors in the human fetal müllerian tract. Am J Obstet Gynecol. (1994) 170:152-6. doi: 10.1016/S0002-9378(13)70297-7

31. Lobie PE, Breipohl W, García Aragón J, Watesr MJ. Cellular localization of the growth hormone receptor/ binding protein in the male and female reproductive systems. Endocrinology. (1990) 126:2214-21. doi: 10.1210/endo-126-4-2214

32. Ferrara N. Vascular endothelial growth factor and the regulation of angiogenesis. Recent Prog Horm Res. (2000) 55:15-35.

33. Hannan NJ, Paiva P, Meehan KL, Rombauts LJF, Gardner DK, Salamonsen LA. Analysis of fertility-related soluble mediators in human uterine fluid identifies VEGF as a key regulator of embryo implantation. Endocrinology. (2011) 152:4948-56. doi: 10.1210/en.2011-1248

34. Kaneko Y, Day ML, Murphy CR. Integrin $\beta 3$ in rat blastocysts and epithelial cells is essential for implantation in vitro: studies with Ishikawa cells and small interfering RNA transfection. Hum Reprod. (2011) 26:1665-74. doi: 10.1093/humrep/der128

35. Lessey BA, Castelbaum AJ. Integrins and implantation in the human. Rev Endocr Metab Disord. (2002) 3:107-17. doi: 10.1023/A:1015450727580

36. Tei C, Maruyama T, Kuji N, Miyazaki T, Mikami M, Yoshimura Y. Reduced expression of alphavbeta3 integrin in the endometrium of unexplained infertility patients with recurrent IVF-ET failures: improvement by danazol treatment. J Assist Reprod Genet. (2003) 20:13-20. doi: 10.1023/A:1021254620888
37. Juengel JL, Nett TM, Anthony R V, Niswender GD. Effects of luteotrophic and luteolytic hormones on expression of mRNA encoding insulin-like growth factor I and growth hormone receptor in the ovine corpus luteum. J Reprod Fertil. (1997) 110:291-8. doi: 10.1530/jrf.0.1100291

38. Schams D, Berisha B, Kosmann M, Einspanier R, Amselgruber WM. Possible role of growth hormone, IGFs, and IGF-binding proteins in the regulation of ovarian function in large farm animals. Domest Anim Endocrinol. (1999) 17:279-85. doi: 10.1016/S0739-7240(99)00044-2

39. Poretsky L, Cataldo NA, Rosenwaks Z, Giudice LC. The insulin-related ovarian regulatory system in health and disease. Endocr Rev. (1999) 20:535-82. doi: 10.1210/edrv.20.4.0374

40. Lanzone A, Di Simone N, Castellani R, Fulghesu AM, Caruso A, Mancuso S. Human growth hormone enhances progesterone production by human luteal cells in vitro: evidence of a synergistic effect with human chorionic gonadotropin. Fertil Steril. (1992) 57:92-6. doi: 10.1016/S0015-0282(16)54782-7

41. Devoto L, Kohen P, Castro O, Vega M, Troncoso JL, Charreau E. Multihormonal regulation of progesterone synthesis in cultured human midluteal cells. J Clin Endocrinol Metab. (1995) 80:1566-70. doi: 10.1210/jcem.80.5.7745001

42. Taketani T, Yamagata Y, Takasaki A, Matsuoka A, Tamura H, Sugino N. Effects of growth hormone and insulin-like growth factor 1 on progesterone production in human luteinized granulosa cells. Fertil Steril. (2008) 90:744-8. doi: 10.1016/j.fertnstert.2007.07.1304

43. Wu H, Li L, Li M, Yuan H. Effect of growth hormone on the endometrial and endometrial blood flow in forzen thawed embryo tranfer. J Reprod Med. (2013) 12:914-917.

44. Carter-Su C, Schwartz J, Argetsinger LS. Growth hormone signaling pathways. Growth Horm IGF Res. (2016) 28:11-5. doi: 10.1016/j.ghir.2015.09.002

45. Conway-Campbell BL, Wooh JW, Brooks AJ, Gordon D, Brown RJ, Lichanska $A M$, et al. Nuclear targeting of the growth hormone receptor results in dysregulation of cell proliferation and tumorigenesis. Proc Natl Acad Sci USA. (2007) 104:13331-6. doi: 10.1073/pnas.0600181104

46. Homburg R, Eshel A, Abdalla HI, Jacobs HS. Growth hormone facilitates ovulation induction by gonadotrophins. Clin Endocrinol (Oxf). (1988) 29:113-7. doi: 10.1111/j.1365-2265.1988.tb00252.x

47. Owen EJ, Shoham Z, Mason BA, Ostergaard H, Jacobs HS. Cotreatment with growth hormone, after pituitary suppression, for ovarian stimulation in in vitro fertilization: a randomized, double-blind, placebo-control trial. Fertil Steril. (1991) 56:1104-10. doi: 10.1016/S0015-0282(16)54724-4

48. Homburg R, Singh A, Bhide P, Shah A, Gudi A. The re-growth of growth hormone in fertility treatment: a critical review. Hum Fertil. (2012) 15:190-3. doi: 10.3109/14647273.2012.744855

49. Eftekhar M, Aflatoonian A, Mohammadian F, Eftekhar T. Adjuvant growth hormone therapy in antagonist protocol in poor responders undergoing assisted reproductive technology. Arch Gynecol Obstet. (2013) 287:1017-21. doi: 10.1007/s00404-012-2655-1

50. Bayoumi YA, Dakhly DMR, Bassiouny YA, Hashish NM. Addition of growth hormone to the microflare stimulation protocol among women with poor ovarian response. Int J Gynecol Obstet. (2015) 131:305-8. doi: 10.1016/j.ijgo.2015.05.034

51. Dakhly DMR, Bayoumi YA, Gad Allah SH. Which is the best IVF/ICSI protocol to be used in poor responders receiving growth hormone as an adjuvant treatment? A prospective randomized trial. Gynecol Endocrinol. (2016) 32:116-9. doi: 10.3109/09513590.2015.1092136

52. Choe SA, Kim MJ, Lee HJ, Kim J, Chang EM, Kim JW, et al. Increased proportion of mature oocytes with sustained-release growth hormone treatment in poor responders: a prospective randomized controlled study. Arch Gynecol Obstet. (2018) 297:791-6. doi: 10.1007/s00404-017-4613-4

53. Dakhly DMR, Bassiouny YA, Bayoumi YA, Hassan MA, Gouda HM, Hassan AA. The addition of growth hormone adjuvant therapy to the long down regulation protocol in poor responders undergoing in vitro fertilization: Randomized control trial. Eur J Obstet Gynecol Reprod Biol. (2018) 228:161-5. doi: 10.1016/j.ejogrb.2018.06.035

54. Yu H, Gao S, Tang H, Chen H, Deng Z, Yang L, et al. Growth hormone intrauterine perfusion combined with replacement cycle in the treatment of non-response thin endometrium: report of 5 cases. Int J Clin Exp Med. (2016) 9:11982-9. 
55. Drakopoulos P, Pluchino N, Bischof P, Cantero P, Meyer P, Chardonnens D. Effect of growth hormone on endometrial thickness and fertility outcome in the treatment of women with panhypopituitarism: a case report. J Reprod Med. (2016) 61:78-82.

56. Sebastian-León P, Garrido N, Remohí J, Pellicer A, Diaz-Gimeno P. Asynchronous and pathological windows of implantation: Two causes of recurrent implantation failure. Hum Reprod. (2018) 33:626-35. doi: 10.1093/humrep/dey023

57. Koot YEM, van Hooff SR, Boomsma CM, van Leenen D, Groot Koerkamp MJA, Goddijn M, et al. An endometrial gene expression signature accurately predicts recurrent implantation failure after IVF. Sci Rep. (2016) 6:19411. doi: 10.1038/srep19411

58. Altmäe S, Martinez-Conejero JA, Salumets A, Simon C, Horcajadas JAA, Stavreus-Evers A, et al. Endometrial gene expression analysis at the time of embryo implantation in women with unexplained infertility. Mol Hum Reprod. (2010) 16:178-87. doi: 10.1093/molehr/gap102

59. Richter KS, Bugge KR, Bromer JG, Levy MJ. Relationship between endometrial thickness and embryo implantation, based on 1,294 cycles of in vitro fertilization with transfer of two blastocyst-stage embryos. Fertil Steril. (2007) 87:53-9. doi: 10.1016/j.fertnstert.2006.05.064

60. Abdalla HI, Brooks AA, Johnson MR, Kirkland A, Thomas A, Studd JWW. Endometrial thickness: a predictor of implantation in ovum recipients? Hum Reprod. (1994) 9:363-5. doi: 10.1093/oxfordjournals.humrep.a138509

61. Kasius A, Smit JG, Torrance HL, Eijkemans MJC, Mol BW, Opmeer $\mathrm{BC}$, et al. Endometrial thickness and pregnancy rates after IVF: a systematic review and meta-analysis. Hum Reprod Update. (2014) 20:530-41. doi: 10.1093/humupd/dmu011

62. Miwa I, Tamura H, Takasaki A, Yamagata Y, Shimamura K, Sugino N. Pathophysiologic features of 'thin' endometrium. Fertil Steril. (2009) 91:9981004. doi: 10.1016/j.fertnstert.2008.01.029

63. Chien L-W, Au H-K, Chen P-L, Xiao J, Tzeng C-R. Assessment of uterine receptivity by the endometrial-subendometrial blood flow distribution pattern in women undergoing in vitro fertilization-embryo transfer. Fertil Steril. (2002) 78:245-51. doi: 10.1016/S0015-0282(02) 03223-5

64. Yovich JL, Ye Y, Keane KN. Growth hormone adjuvant trial for poor responders undergoing IVF. Eur J Obstet Gynecol Reprod Biol. (2019) 236:249. doi: 10.1016/j.ejogrb.2019.01.026

65. Jiang $\mathrm{H}$, Pei $\mathrm{H}$, Zhang $\mathrm{W}$, Wang $\mathrm{X}$. A prospective clinical study of interventional ultrasound sclerotherapy on women with hydrosalpinx before in vitro fertilization and embryo transfer. Fertil Steril. (2010) 94:2854-6. doi: 10.1016/j.fertnstert.2010.06.065

66. Spiliotis BE. Growth hormone insufficiency and its impact on ovarian function. Ann N Y Acad Sci. (2003) 997:77-84. doi: 10.1196/annals. 1290.009

67. Altmäe S, Esteban FJ, Stavreus-Evers A, Simón C, Giudice L, Lessey BA, et al. Guidelines for the design, analysis and interpretation of "omics" data: focus on human endometrium. Hum Reprod Update. (2014) 20:12-28. doi: 10.1093/humupd/dmt048

68. Keane KN, Yovich JL, Hamidi A, Hinchliffe PM, Dhaliwal SS. Singlecentre retrospective analysis of growth hormone supplementation in IVF patients classified as poor-prognosis. BMJ Open. (2017) 7:e018107. doi: 10.1136/bmjopen-2017-018107

Conflict of Interest: The authors declare that the research was conducted in the absence of any commercial or financial relationships that could be construed as a potential conflict of interest.

Copyright (c) 2019 Altmäe and Aghajanova. This is an open-access article distributed under the terms of the Creative Commons Attribution License (CC BY). The use, distribution or reproduction in other forums is permitted, provided the original author(s) and the copyright owner(s) are credited and that the original publication in this journal is cited, in accordance with accepted academic practice. No use, distribution or reproduction is permitted which does not comply with these terms. 